\title{
Moduli of regular singular parabolic connections with given spectral type on smooth projective curves
}

\author{
By Michi-aki InABA and Masa-Hiko SaIto
}

(Received Nov. 6, 2016)

\begin{abstract}
We define a moduli space of stable regular singular parabolic connections with given spectral type on smooth projective curves and show the smoothness of the moduli space and give a relative symplectic structure on the moduli space. Moreover, we define the isomonodromic deformation on this moduli space and prove the geometric Painlevé property of the isomonodromic deformation.
\end{abstract}

\section{Introduction.}

Let $T$ be a smooth covering of the moduli stack of $n$-pointed smooth projective curves of genus $g$. Take a universal family $(\mathcal{C}, \tilde{\boldsymbol{t}})$ over $T$. In the paper [4], the first author constructed the relative moduli space

$$
M_{\mathcal{C} / T}^{\boldsymbol{\alpha}}(\tilde{\boldsymbol{t}}, r, d) \longrightarrow T \times \Lambda_{r}^{(n)}(d)
$$

of regular singular $\boldsymbol{\alpha}$-stable parabolic connections of rank $r$ and degree $d$ on $\mathcal{C} / T$. Here $\boldsymbol{\alpha}=\left(\alpha_{j}^{(i)}\right)_{1 \leq i \leq n}^{1 \leq i \leq n}$ are rational numbers such that $0<\alpha_{1}^{(i)}<\cdots<\alpha_{r}^{(i)}<1$ and that $\alpha_{j}^{(i)} \neq \alpha_{j^{\prime}}^{\left(i^{\prime}\right)}$ for any $(i, j) \neq\left(i^{\prime}, j^{\prime}\right) . \Lambda_{r}^{(n)}(d)$ is given by

$$
\Lambda_{r}^{(n)}(d):=\left\{\left(\lambda_{j}^{(i)}\right)_{0 \leq j \leq r-1}^{\substack{1 \leq i \leq n \\ 0 \leq j \leq \mathbb{C}^{n r}}} \mid d+\sum_{i=1}^{n} \sum_{j=0}^{r-1} \lambda_{j}^{(i)}=0\right\} .
$$

Then for any point $(x, \boldsymbol{\lambda}) \in T \times \Lambda_{r}^{(n)}(d)$, the fiber $M_{\mathcal{C} / T}^{\boldsymbol{\alpha}}(\tilde{\boldsymbol{t}}, r, d)_{(x, \boldsymbol{\lambda})}$ is smooth of dimension $2 r^{2}(g-1)+n r(r-1)+2$. He also constructed the algebraic splitting

$$
D: \pi^{*}\left(\Theta_{T}\right) \longrightarrow \Theta_{M_{\mathcal{C} / T}^{\alpha}(\tilde{\boldsymbol{t}}, r, d)}
$$

of the canonical surjection $\Theta_{M_{\mathcal{C} / T}^{\boldsymbol{\alpha}}(\tilde{\boldsymbol{t}}, r, d)} \rightarrow \pi^{*}\left(\Theta_{T}\right)$, where $\pi: M_{\mathcal{C} / T}^{\boldsymbol{\alpha}}(\tilde{\boldsymbol{t}}, r, d) \rightarrow T$ is the structure morphism. The subbundle $D\left(\pi^{*}\left(\Theta_{T}\right)\right) \subset \Theta_{M_{\mathcal{C} / T}^{\alpha}(\tilde{\boldsymbol{t}}, r, d)}$ satisfies the integrability condition and the associated foliation $\mathcal{F}_{M_{\mathcal{C} / T}^{\alpha}(\tilde{\boldsymbol{t}}, r, d)}$ is nothing but the isomonodromic

2010 Mathematics Subject Classification. Primary 14D20; Secondary 34M56, 34M55.

Key Words and Phrases. regular singular connection of spectral type, moduli space of parabolic connections, symplectic structure, Riemann-Hilbert correspondence, geometric Painlevé property, isomonodromic deformation of linear connection, higher dimensional Painlevé equations.

This work was partly supported by JSPS KAKENHI: Grant Numbers JP17H06127, JP15K13427, JP24224001, JP22740014, JP26400043. 
deformation. One of the important results in [4] is that the isomonodromic deformation determined by $D\left(\pi^{*}\left(\Theta_{T}\right)\right)$ has the geometric Painlevé property.

There is a locus $Y$ in $M_{\mathcal{C} / T}^{\boldsymbol{\alpha}}(\tilde{\boldsymbol{t}}, r, d)$ such that $\left(E, \nabla,\left\{l_{j}^{(i)}\right\}\right) \in M_{\mathcal{C} / T}^{\boldsymbol{\alpha}}(\tilde{\boldsymbol{t}}, r, d)$ lies in $Y$ if and only if the residue matrix of $\nabla$ at $t_{i}$ is given by

$$
(\dagger) \quad\left(\begin{array}{cccc}
\mu_{1}^{(i)} I_{r_{s_{i}-1}} & * & * & * \\
0 & \mu_{2}^{(i)} I_{r_{s_{i}-2}} & * & * \\
\vdots & \vdots & \ddots & \vdots \\
0 & 0 & \cdots & \mu_{s_{i}}^{(i)} I_{r_{0}}
\end{array}\right) \text {. }
$$

We can easily see that the locus $Y$ is preserved by the isomonodromic deformation. However, the dimension of $Y$ is too big because it parameterizes the parabolic structure $\left\{l_{j}^{(i)}\right\}$. So we contract $Y$ by forgetting the data $\left\{l_{j}^{(i)}\right\}$ and obtain a moduli space $\bar{Y}$. We say $\bar{Y}$ the moduli space of regular singular parabolic connections of spectral type $(\dagger)$. By construction, $\bar{Y}$ is preserved by the isomonodromic deformation. So we obtain a low dimensional phase space arising from the isomonodromic deformation. Such low dimensional phase spaces get an attention from the viewpoint of the theory of integrable systems.

Oshima studied in [9] the isomonodromic deformation of the Fuchsian system of spectral types in detail. In particular, he studied additive Deligne-Simpson problem on Fuchsian systems on trivial bundles on $\mathbf{P}^{1}$ and a combinatorial structure of middle convolutions and their relation to a Kac-Mooody root system discovered by CrawleyBoevey [1].

Let us fix a smooth projective curve $C$ of genus $g$ and a set of $n$-distinct points $\boldsymbol{t}=\left(t_{1}, \cdots, t_{n}\right)$ on $C$. Spectral types are given by tuples $\left(r_{j}^{(i)}\right)_{\substack{1 \leq i \leq n \\ 0 \leq j \leq s_{i}-1}}$ of partitions of integers, where $r$ is a fixed rank of vector bundles and at each singular point $t_{i}$, $r_{j}^{(i)}$ are positive integers such that $\sum_{j=0}^{s_{i}-1} r_{j}^{(i)}=r$. Fixing a degree $d$ and a spectral type $\left(r_{j}^{(i)}\right)_{0 \leq j \leq s_{i}-1}^{\substack{1 \leq i \leq n \\ 0 \leq j \leq}}$, let us take any local exponents $\boldsymbol{\nu} \in N\left(d,\left(r_{j}^{(i)}\right)^{1 \leq i \leq n}\right)$ (see Section 4). Then we can define the moduli space $M^{\boldsymbol{\alpha}}\left(C, \boldsymbol{t}, \boldsymbol{\nu}, d,\left(r_{j}^{(i)}\right)\right)$ of $\boldsymbol{\alpha}$-stable $\boldsymbol{\nu}$-parabolic connections on $(C, \boldsymbol{t})$ of spectral type $\left(r_{j}^{(i)}\right)$. In Section 1 , we show that $M^{\boldsymbol{\alpha}}\left(C, \boldsymbol{t}, \boldsymbol{\nu}, d,\left(r_{j}^{(i)}\right)\right)$ is a smooth quasi-projective scheme of dimension (see Theorem 1.3)

$$
\operatorname{dim} M^{\boldsymbol{\alpha}}\left(C, \boldsymbol{t}, \boldsymbol{\nu}, d,\left(r_{j}^{(i)}\right)\right)=2 r^{2}(g-1)+2+2 \sum_{i=1}^{n} \sum_{j=0}^{s_{i}-1} \sum_{j^{\prime}>j} r_{j}^{(i)} r_{j^{\prime}}^{(i)} .
$$

If we set

$$
N=r^{2}(g-1)+1+n \frac{r(r-1)}{2}
$$

one can rewrite as

$$
\operatorname{dim} M^{\boldsymbol{\alpha}}\left(C, \boldsymbol{t}, \boldsymbol{\nu}, d,\left(r_{j}^{(i)}\right)\right)=2\left(N-\sum_{i=1}^{n} \sum_{j=0}^{s_{i}-1} \frac{r_{j}^{(i)}\left(r_{j}^{(i)}-1\right)}{2}\right) .
$$


The moduli space of $\boldsymbol{\alpha}$-stable parabolic connections of spectral types $\left(r_{j}^{(i)}\right)$ is a deformation of the moduli space of $\boldsymbol{\alpha}$-stable parabolic Higgs bundles on $(C, \boldsymbol{t})$ of spectral types $\left(r_{j}^{(i)}\right)$. Then the genus of spectral curves of parabolic Higgs bundles should be the half of dimension of the moduli spaces. The formula suggests that the genus of spectral curves equal to $N-\sum_{i=1}^{n} \sum_{j=0}^{s_{i}-1} r_{j}^{(i)}\left(r_{j}^{(i)}-1\right) / 2$ where $N$ is the genus of spectral curve with trivial spectral types $r_{j}^{(i)}=1$. It will be interesting to see the explicit geometry of the moduli space of parabolic connections and parabolic Higgs bundles. An approach by using the apparent singularities and their duals will be treated in [10].

For example, if we consider the case $g=0, n=4, r=2, d=-1$ and $r_{j}^{(i)}=1$ for all $i, j$, then the spectral type will be denoted as $(11,11,11,11)$. The corresponding moduli spaces $M$ are nothing but the fiber of the phase space, or Okamoto's space of initial conditions of Painlevé VI equations and $\operatorname{dim} M=2$.

Yamakawa constructed in [13] the moduli space of stable filtered local systems and it is analytically isomorphic to the moduli space $M^{\boldsymbol{\alpha}}\left(C, \boldsymbol{t}, \boldsymbol{\nu}, d,\left(r_{j}^{(i)}\right)\right)$ under the RiemannHilbert morphism.

Sakai studied in $[\mathbf{1 1}]$ the Fuchsian system of spectral type which gives 4-dimensional isomonodromic deformation equations. Here the 4-dimensional means that the dimension of the moduli space of parabolic connection of spectral type is 4 . The interesting point of $[\mathbf{1 1}]$ is that a Fuji-Suzuki system $([\mathbf{2}],[\mathbf{3}])$ and a Sasano system $([\mathbf{1 2}])$ can be obtained from the isomonodromic deformations of the Fuchsian system of certain spectral types. Including them, there exists only 4-types of 4-dimensional isomonodromic deformation equations of Fuchsian systems of spectral types over $\mathbf{P}^{1}$. They are corresponding to the spectral types $r=2, n=5,(11,11,11,11,11)$ (Garnier), $r=3, n=4,(21,21,111,111)$ (the Fuji-Suzuki), $r=4, n=4,(31,22,22,1111)$ (Sasano) and $r=4, n=4,(22,22,22,211)$ (the sixth matrix Painlevé).

The main results in this paper are the smoothness and a symplectic structure of the moduli space of stable regular singular parabolic connections of any spectral type on smooth projective curves over $\mathbb{C}$. (Cf. Theorem 1.2 and Theorem 3.1). Moreover, the more important result (cf. Theorem 4.1) is that the isomonodromic deformation defined on the moduli space of regular singular parabolic connections of spectral type has the geometric Painlevé property. So we can say that the moduli space of stable regular singular parabolic connections with given spectral type is the space of initial conditions for the isomonodromic deformations.

Here the definition of the geometric Painleve property is given in [6] and the geometric Painlevé property implies the usual Painlevé property.

As a corollary, 4-dimensional isomonodromic deformation considered by Sakai in [11] has the Painlevé property.

It will be also interesting to consider similar problems for parabolic connections with irregular singularities of fixing spectral types. Classifications of spectral types of dimension 4 cases are treated in $[\mathbf{7}]$ and $[8]$. 


\section{Definition and properties of the moduli space of regular singular par- abolic connections with given spectral type.}

Let $C$ be a smooth projective irreducible curve over $\mathbb{C}$ of genus $g$. We set

$$
T_{n}:=\left\{\boldsymbol{t}=\left(t_{1}, \ldots, t_{n}\right) \in C \times \cdots \times C \mid t_{i} \neq t_{j} \text { for } i \neq j\right\} .
$$

Let $r, d$ be integers with $r>0$. For each $i$ with $1 \leq i \leq n$, take positive integers $r_{0}^{(i)}, \ldots, r_{s_{i}-1}^{(i)}$ such that $r=\sum_{j=0}^{s_{i}-1} r_{j}^{(i)}$ for any $i$. Set

$$
N\left(d,\left(r_{j}^{(i)}\right)\right):=\left\{\begin{array}{l|l}
\left(\nu_{j}^{(i)}\right)_{\substack{1 \leq i \leq n \\
0 \leq j \leq s_{i}-1}} \mid \begin{array}{l}
\nu_{j}^{(i)} \in \mathbb{C} \text { for any } i, j \text { and } \\
d+\sum_{i=1}^{n} \sum_{j=0}^{s_{i}-1} r_{j}^{(i)} \nu_{j}^{(i)}=0
\end{array}
\end{array}\right\} .
$$

Definition 1.1. Take $\boldsymbol{t} \in T_{n}$ and $\boldsymbol{\nu}=\left(\nu_{j}^{(i)}\right) \in N\left(d,\left(r_{j}^{(i)}\right)\right)$. We say $\left(E, \nabla,\left\{l_{j}^{(i)}\right\}\right)$ is a regular singular $(\boldsymbol{t}, \boldsymbol{\nu})$-parabolic connection of spectral type $\left(r_{j}^{(i)}\right)_{\substack{1 \leq i \leq n \\ 0 \leq j \leq s_{i}-1}}$ if

(1) $E$ is an algebraic vector bundle on $C$ of rank $r$ and degree $d$,

(2) $\nabla: E \longrightarrow E \otimes \Omega_{C}^{1}\left(t_{1}+\cdots+t_{n}\right)$ is a connection,

(3) for each $i,\left.E\right|_{t_{i}}=l_{0}^{(i)} \supset l_{1}^{(i)} \supset \cdots \supset l_{s_{i}-1}^{(i)} \supset l_{s_{i}}^{(i)}=0$ is a filtration such that $\operatorname{dim}_{\mathbb{C}}\left(l_{j}^{(i)} / l_{j+1}^{(i)}\right)=r_{j}^{(i)}$ and

(4) $\left(\operatorname{res}_{t_{i}}(\nabla)-\nu_{j}^{(i)} \mathrm{id}\right)\left(l_{j}^{(i)}\right) \subset l_{j+1}^{(i)}$ for any $i, j$.

Take rational numbers $\boldsymbol{\alpha}=\left(\alpha_{j}^{(i)}\right)_{\substack{1 \leq i \leq n \\ 1 \leq s_{i}}}$ such that $0<\alpha_{1}^{(i)}<\alpha_{2}^{(i)}<\cdots<\alpha_{s_{i}}^{(i)}<1$ for any $i, j$ and $\alpha_{j}^{(i)} \neq \alpha_{j^{\prime}}^{\left(i^{\prime}\right)}$ for $(i, j) \neq\left(i^{\prime}, j^{\prime}\right)$.

Definition 1.2. A regular singular $(\boldsymbol{t}, \boldsymbol{\nu})$-parabolic connection $\left(E, \nabla,\left\{l_{j}^{(i)}\right\}\right)$ of spectral type $\left(r_{j}^{(i)}\right)$ is said to be $\boldsymbol{\alpha}$-stable (resp. $\boldsymbol{\alpha}$-semistable) if

$$
\begin{gathered}
\frac{\operatorname{deg} F+\sum_{i=1}^{n} \sum_{j=1}^{s_{i}} \alpha_{j}^{(i)} \operatorname{dim}_{\mathbb{C}}\left(\left(\left.F\right|_{t_{i}} \cap l_{j-1}^{(i)}\right) /\left(\left.F\right|_{t_{i}} \cap l_{j}^{(i)}\right)\right)}{\operatorname{rank} F} \\
\quad< \\
(\text { resp. } \leq)
\end{gathered}
$$

for any subbundle $0 \neq F \subsetneq E$ with $\nabla(F) \subset F \otimes \Omega_{C}^{1}\left(t_{1}+\cdots+t_{n}\right)$.

Let $T$ be a smooth algebraic scheme which is a smooth covering of the moduli stack of $n$-pointed smooth projective irreducible curves of genus $g$ over $\mathbb{C}$ and $(\mathcal{C}, \tilde{\boldsymbol{t}})$ be the universal family over $T\left(\tilde{\boldsymbol{t}}=\left(\tilde{t}_{1}, \ldots, \tilde{t}_{n}\right)\right.$, where each $\tilde{t}_{i}$ is a section of $\mathcal{C} \rightarrow T$ and $\tilde{t}_{i} \cap \tilde{t}_{j}=\emptyset$ for any $\left.i \neq j\right)$.

TheOREM 1.1. There exists a relative coarse moduli scheme $\pi: M_{\mathcal{C} / T}^{\alpha}\left(d,\left(r_{j}^{(i)}\right)\right) \rightarrow$ $T \times N\left(d,\left(r_{j}^{(i)}\right)\right)$ of $\boldsymbol{\alpha}$-stable regular singular parabolic connections of spectral type $\left(r_{j}^{(i)}\right)$. Moreover $\pi$ is a quasi-projective morphism. 
Proof. Proof is the same as that of [4, Theorem 2.1], which essentially uses [5, Theorem 5.1] and we omit the proof here.

THEOREM 1.2. The relative moduli space $\pi: M_{\mathcal{C} / T}^{\boldsymbol{\alpha}}\left(d,\left(r_{j}^{(i)}\right)\right) \rightarrow T \times N\left(d,\left(r_{j}^{(i)}\right)\right)$ is smooth.

Proof. Let $M_{\mathcal{C} / T}(d,(1))$ be the moduli space of pairs $\left(L, \nabla_{L}\right)$ of a line bundle $L$ on $\mathcal{C}_{x}$ and a connection $\nabla_{L}: L \rightarrow L \otimes \Omega_{\mathcal{C} / T}^{1}\left(\tilde{t}_{1}+\cdots+\tilde{t}_{n}\right)$. Then $M_{\mathcal{C} / T}(d,(1))$ is an affine space bundle over $\operatorname{Pic}_{\mathcal{C} / T}^{d} \times N(d,(1))$, where

$$
N(d,(1)):=\left\{\left(\nu^{(i)}\right) \in \mathbb{C}^{n} \mid d+\sum_{i=1}^{n} \nu^{(i)}=0\right\} .
$$

Since $\operatorname{Pic}_{\mathcal{C} / T}^{d}$ is smooth over $T, M_{\mathcal{C} / T}(d,(1))$ is smooth over $T \times N(d,(1))$. Consider the morphism

$$
\begin{gathered}
\operatorname{det}: M_{\mathcal{C} / T}^{\boldsymbol{\alpha}}\left(d,\left(r_{j}^{(i)}\right)\right) \longrightarrow M_{\mathcal{C} / T}(d,(1)) \times_{N(d,(1))} N\left(d,\left(r_{j}^{(i)}\right)\right) \\
\left(E, \nabla,\left\{l_{j}^{(i)}\right\}\right) \mapsto\left((\operatorname{det}(E), \operatorname{det}(\nabla)), \pi\left(E, \nabla,\left\{l_{j}^{(i)}\right\}\right)\right) .
\end{gathered}
$$

It is sufficient to show that the morphism det is smooth. Let $A$ be an Artinian local ring over $M_{\mathcal{C} / T}(d,(1)) \times_{N(d,(1))} N\left(d,\left(r_{j}^{(i)}\right)\right)$ with the maximal ideal $m$ and $I$ be an ideal of $A$ such that $m I=0$. Let $\left(L, \nabla_{L}\right) \in M_{\mathcal{C} / T}(d,(1))(A)$ and $\nu=\left(\nu_{j}^{(i)}\right) \in N\left(d,\left(r_{j}^{(i)}\right)\right)(A)$ be the elements corresponding to the morphism $\operatorname{Spec} A \rightarrow M_{\mathcal{C} / T}(d,(1)) \times_{N(d,(1))} N\left(d,\left(r_{j}^{(i)}\right)\right)$. Take any member $\left(E, \nabla,\left\{l_{j}^{(i)}\right\}\right) \in \mathcal{M}_{\mathcal{C} / T}^{\alpha}\left(d,\left(r_{j}^{(i)}\right)\right)(A / I)$ such that $\left(\operatorname{res}_{\tilde{t}_{i} \times A / I}(\nabla)-\right.$ $\left.\nu_{j}^{(i)} \mathrm{id}\right)\left(l_{j}^{(i)}\right) \subset l_{j+1}^{(i)}$ for any $i, j$ and that $\operatorname{det}\left(E, \nabla,\left\{l_{j}^{(i)}\right\}\right) \cong\left(\left(L, \nabla_{L}\right), \nu\right) \otimes A / I$. It is sufficient to show that $\left(E, \nabla,\left\{l_{j}^{(i)}\right\}\right)$ can be lifted to a flat family $\left(\tilde{E}, \tilde{\nabla},\left\{\tilde{l}_{j}^{(i)}\right\}\right)$ over $A$ such that $\operatorname{det}\left(\tilde{E}, \tilde{\nabla},\left\{\tilde{l}_{j}^{(i)}\right\}\right) \cong\left(\left(L, \nabla_{L}\right), \boldsymbol{\nu}\right)$. We define a complex $\mathcal{F}_{0}^{\bullet}$ by

$\mathcal{F}_{0}^{0}:=\left\{a \in \mathcal{E} n d(E \otimes A / m) \mid \operatorname{Tr}(a)=0\right.$ and $\left.a\right|_{\tilde{t}_{i} \times A / m}\left(\left(l_{j}^{(i)}\right)_{A / m}\right) \subset\left(l_{j}^{(i)}\right)_{A / m}$ for any $\left.i, j\right\}$

$\mathcal{F}_{0}^{1}:=\left\{\begin{array}{l|l}b \in \mathcal{E} n d(E \otimes A / m) \otimes \Omega_{\mathcal{C} / T}^{1}\left(\tilde{t}_{1}+\cdots+\tilde{t}_{n}\right) & \begin{array}{l}\operatorname{Tr}(b)=0 \text { and } \\ \operatorname{res}_{\tilde{t}_{i} \otimes A / m}(b)\left(\left(l_{j}^{(i)}\right)_{A / m}\right) \subset\left(l_{j+1}^{(i)}\right)_{A / m} \\ \text { for any } i, j\end{array}\end{array}\right\}$ $\nabla^{\dagger}: \mathcal{F}_{0}^{0} \ni a \mapsto \nabla \circ a-a \circ \nabla \in \mathcal{F}_{0}^{1}$.

Let $\mathcal{C}_{A}=\bigcup_{\alpha} U_{\alpha}$ be an affine open covering such that $\left.E\right|_{U_{\alpha} \otimes A / I} \cong \mathcal{O}_{U_{\alpha} \otimes A / I}^{\oplus r}$, $\sharp\left\{\left(\tilde{t}_{i}\right)_{A} \mid\left(\tilde{t}_{i}\right)_{A} \in U_{\alpha}\right\} \leq 1$ for any $\alpha$ and $\sharp\left\{\alpha \mid\left(\tilde{t}_{i}\right)_{A} \in U_{\alpha}\right\}=1$ for any $i$. Take a free $\mathcal{O}_{U_{\alpha}}$-module $E_{\alpha}$ of rank $r$ with isomorphisms $\varphi_{\alpha}:\left.\operatorname{det}\left(E_{\alpha}\right) \stackrel{\sim}{\rightarrow} L\right|_{U_{\alpha}}$ and $\phi_{\alpha}: E_{\alpha} \otimes A / I \stackrel{\sim}{\rightarrow}$ $\left.E\right|_{U_{\alpha} \otimes A / I}$ such that

$$
\varphi_{\alpha} \otimes A / I=\operatorname{det}\left(\phi_{\alpha}\right):\left.\operatorname{det}\left(E_{\alpha}\right) \stackrel{\sim}{\longrightarrow} \operatorname{det}(E)\right|_{U_{\alpha} \otimes A / I}=\left.(L \otimes A / I)\right|_{U_{\alpha} \otimes A / I} .
$$

If $\left(\tilde{t}_{i}\right)_{A} \in U_{\alpha}$, we may assume that the parabolic structure $\left\{l_{j}^{(i)}\right\}$ is given by 


$$
l_{j}^{(i)}=\left\langle\left. e_{1}\right|_{\left(\tilde{t}_{i}\right)_{A / I}}, \ldots,\left.e_{r_{j}^{(i)}+\cdots+r_{s_{i}-1}^{(i)}}\right|_{\left(\tilde{t}_{i}\right)_{A / I}}\right\rangle
$$

where $e_{1}, \ldots, e_{r}$ is the standard basis of $E_{\alpha}$. We define a parabolic structure $\left\{\left(l_{\alpha}\right)_{j}^{(i)}\right\}$ on $E_{\alpha}$ by

$$
\left(l_{\alpha}\right)_{j}^{(i)}:=\left\langle\left. e_{1}\right|_{\left(\tilde{t}_{i}\right)_{A}}, \ldots,\left.e_{r_{j}^{(i)}+\cdots+r_{s_{i}-1}^{(i)}}\right|_{\left(\tilde{t}_{i}\right)_{A}}\right\rangle .
$$

The connection $\phi_{\alpha}^{-1} \circ\left(\left.\nabla\right|_{U_{\alpha}}\right) \circ \phi_{\alpha}: E_{\alpha} \otimes A / I \rightarrow E_{\alpha} \otimes \Omega_{\mathcal{C} / T}^{1}\left(\tilde{t}_{1}+\cdots+\tilde{t}_{n}\right) \otimes A / I$ is given by a connection matrix $\bar{B}_{\alpha} \in H^{0}\left(E_{\alpha}^{\vee} \otimes E_{\alpha} \otimes \Omega_{\mathcal{C} / T}^{1}\left(\tilde{t}_{1}+\cdots+\tilde{t}_{n}\right) \otimes A / I\right)$. Then we have

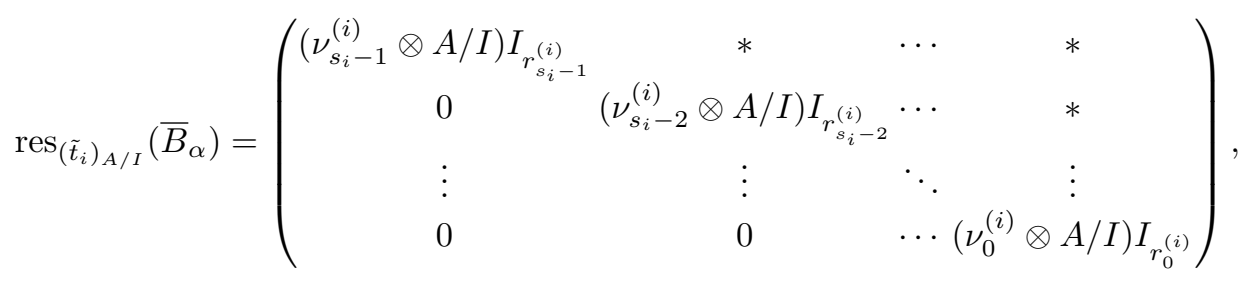

where $I_{r_{j}^{(i)}}$ is the identity $r_{j}^{(i)} \times r_{j}^{(i)}$ matrix. We can take a lift $B_{\alpha} \in H^{0}\left(E_{\alpha}^{\vee} \otimes E_{\alpha} \otimes\right.$ $\left.\Omega_{\mathcal{C} / T}^{1}\left(\tilde{t}_{1}+\cdots+\tilde{t}_{n}\right)\right)$ of $\bar{B}_{\alpha}$ such that

$$
\operatorname{res}_{\left(\tilde{t}_{i}\right)_{A}}\left(B_{\alpha}\right)=\left(\begin{array}{ccccc}
\nu_{s_{i}-1}^{(i)} I_{r_{s_{i}-1}^{(i)}} & * & \cdots & * \\
0 & \nu_{s_{i}-2}^{(i)} I_{r_{s_{i}-2}^{(i)}} & \cdots & * \\
\vdots & \vdots & \ddots & \vdots \\
0 & 0 & \cdots & \nu_{0}^{(i)} I_{r_{0}^{(i)}}
\end{array}\right)
$$

and that $\operatorname{Tr}\left(B_{\alpha}\right)\left(e_{1} \wedge \cdots \wedge e_{r}\right)=\left(\varphi_{\alpha} \otimes \mathrm{id}\right)^{-1}\left(\left.\nabla_{L}\right|_{U_{\alpha}}\left(\varphi_{\alpha}\left(e_{1} \wedge \cdots \wedge e_{r}\right)\right)\right)$. Consider the connection $\nabla_{\alpha}: E_{\alpha} \rightarrow E_{\alpha} \otimes \Omega_{\mathcal{C} / T}^{1}\left(\tilde{t}_{1}+\cdots+\tilde{t}_{n}\right)$ defined by

$$
\nabla\left(\begin{array}{c}
f_{1} \\
\vdots \\
f_{r}
\end{array}\right)=\left(\begin{array}{c}
d f_{1} \\
\vdots \\
d f_{r}
\end{array}\right)+B_{\alpha}\left(\begin{array}{c}
f_{1} \\
\vdots \\
f_{r}
\end{array}\right) \quad\left(\left(\begin{array}{c}
f_{1} \\
\vdots \\
f_{r}
\end{array}\right) \in E_{\alpha}\right)
$$

Then we obtain a local parabolic connection $\left(E_{\alpha}, \nabla_{\alpha},\left\{\left(l_{\alpha}\right)_{j}^{(i)}\right\}\right)$ on $U_{\alpha}$. If $\left(\tilde{t}_{i}\right)_{A} \notin U_{\alpha}$ for any $i$, then we can easily obtain a local parabolic connection $\left(E_{\alpha}, \nabla_{\alpha},\left\{\left(l_{\alpha}\right)_{j}^{(i)}\right\}\right)$ on $U_{\alpha}$ (in this case, a parabolic structure $\left\{\left(l_{\alpha}\right)_{j}^{(i)}\right\}$ is nothing). We put $U_{\alpha \beta}:=U_{\alpha} \cap U_{\beta}$ and $U_{\alpha \beta \gamma}:=U_{\alpha} \cap U_{\beta} \cap U_{\gamma}$. Take an isomorphism

$$
\theta_{\beta \alpha}:\left.\left.E_{\alpha}\right|_{U_{\alpha \beta}} \stackrel{\sim}{\longrightarrow} E_{\beta}\right|_{U_{\alpha \beta}}
$$

such that $\theta_{\beta \alpha} \otimes A / I=\phi_{\beta}^{-1} \circ \phi_{\alpha}$ and that $\varphi_{\beta} \circ \operatorname{det}\left(\theta_{\beta \alpha}\right)=\varphi_{\alpha}$. We put

$$
u_{\alpha \beta \gamma}:=\phi_{\alpha} \circ\left(\left.\left.\left.\theta_{\gamma \alpha}^{-1}\right|_{U_{\alpha \beta \gamma}} \circ \theta_{\gamma \beta}\right|_{U_{\alpha \beta \gamma}} \circ \theta_{\beta \alpha}\right|_{U_{\alpha \beta \gamma}}-\mathrm{id}_{\left.E_{\alpha}\right|_{U_{\alpha \beta \gamma}}}\right) \circ \phi_{\alpha}^{-1}
$$


and

$$
v_{\alpha \beta}:=\phi_{\alpha} \circ\left(\left.\nabla_{\alpha}\right|_{U_{\alpha \beta}}-\left.\theta_{\beta \alpha}^{-1} \circ \nabla_{\beta}\right|_{U_{\alpha \beta}} \circ \theta_{\beta \alpha}\right) \circ \phi_{\alpha}^{-1} .
$$

Then we have $\left\{u_{\alpha \beta \gamma}\right\} \in C^{2}\left(\left\{U_{\alpha}\right\}, \mathcal{F}_{0}^{0} \otimes I\right)$ and $\left\{v_{\alpha \beta}\right\} \in C^{1}\left(\left\{U_{\alpha}\right\}, \mathcal{F}_{0}^{1} \otimes I\right)$. We can easily see that

$$
d\left\{u_{\alpha \beta \gamma}\right\}=0 \quad \text { and } \quad \nabla^{\dagger}\left\{u_{\alpha \beta \gamma}\right\}=-d\left\{v_{\alpha \beta}\right\} .
$$

So we can define an element

$$
\omega\left(E, \nabla,\left\{l_{j}^{(i)}\right\}\right):=\left[\left\{u_{\alpha \beta \gamma}\right\},\left\{v_{\alpha \beta}\right\}\right] \in \mathbf{H}^{2}\left(\mathcal{F}_{0}^{\bullet}\right) \otimes I .
$$

We can check that $\omega\left(E, \nabla,\left\{l_{j}^{(i)}\right\}\right)=0$ if and only if $\left(E, \nabla,\left\{l_{j}^{(i)}\right\}\right)$ can be lifted to a flat family $\left(\tilde{E}, \tilde{\nabla},\left\{\tilde{l}_{j}^{(i)}\right\}\right)$ over $A$ such that $\operatorname{det}\left(\tilde{E}, \tilde{\nabla},\left\{\tilde{l}_{j}^{(i)}\right\}\right) \cong\left(\left(L, \nabla_{L}\right), \boldsymbol{\nu}\right)$. From the spectral sequence $H^{q}\left(\mathcal{F}_{0}^{p}\right) \Rightarrow \mathbf{H}^{p+q}\left(\mathcal{F}_{0}^{\bullet}\right)$, there is an isomorphism

$$
\mathbf{H}^{2}\left(\mathcal{F}_{0}^{\bullet}\right) \cong \operatorname{coker}\left(H^{1}\left(\mathcal{F}_{0}^{0}\right) \stackrel{H^{1}\left(\nabla^{\dagger}\right)}{\longrightarrow} H^{1}\left(\mathcal{F}_{0}^{1}\right)\right) \text {. }
$$

Since $\left(\mathcal{F}_{0}^{0}\right)^{\vee} \otimes \Omega_{\mathcal{C} / T}^{1} \cong \mathcal{F}_{0}^{1}$ and $\left(\mathcal{F}_{0}^{1}\right)^{\vee} \otimes \Omega_{\mathcal{C} / T}^{1} \cong \mathcal{F}_{0}^{0}$, we have

$$
\begin{aligned}
\mathbf{H}^{2}\left(\mathcal{F}_{0}^{\bullet}\right) & \cong \operatorname{coker}\left(H^{1}\left(\mathcal{F}_{0}^{0}\right) \stackrel{H^{1}\left(\nabla^{\dagger}\right)}{\longrightarrow} H^{1}\left(\mathcal{F}_{0}^{1}\right)\right) \\
& \cong \operatorname{ker}\left(H^{1}\left(\mathcal{F}_{0}^{1}\right)^{\vee} \stackrel{H^{1}\left(\nabla^{\dagger}\right)}{\longrightarrow} H^{1}\left(\mathcal{F}_{0}^{0}\right)^{\vee}\right)^{\vee} \\
& \cong \operatorname{ker}\left(H^{0}\left(\left(\mathcal{F}_{0}^{1}\right)^{\vee} \otimes \Omega_{\mathcal{C} / T}^{1}\right) \stackrel{-H^{0}\left(\nabla^{\dagger}\right)}{\longrightarrow} H^{0}\left(\left(\mathcal{F}_{0}^{0}\right)^{\vee} \otimes \Omega_{\mathcal{C} / T}^{1}\right)\right)^{\vee} \\
& \cong \operatorname{ker}\left(H^{0}\left(\mathcal{F}_{0}^{0}\right) \stackrel{-H^{0}\left(\nabla^{\dagger}\right)}{\longrightarrow} H^{0}\left(\mathcal{F}_{0}^{1}\right)\right)^{\vee} .
\end{aligned}
$$

Take any element $a \in \operatorname{ker}\left(H^{0}\left(\mathcal{F}_{0}^{0}\right) \stackrel{-H^{0}\left(\nabla^{\dagger}\right)}{\longrightarrow} H^{0}\left(\mathcal{F}_{0}^{1}\right)\right)$. Then we have $a \in$ $\operatorname{End}\left(\left(E, \nabla,\left\{l_{j}^{(i)}\right\}\right) \otimes A / m\right)$. Since $\left(E, \nabla,\left\{l_{j}^{(i)}\right\}\right) \otimes A / m$ is $\boldsymbol{\alpha}$-stable, we have $a=c \cdot \operatorname{id}_{E \otimes A / m}$ for some $c \in A / m$. So we have $a=0$, because $\operatorname{Tr}(a)=0$. Thus we have $\operatorname{ker}\left(H^{0}\left(\mathcal{F}_{0}^{0}\right) \stackrel{-H^{0}\left(\nabla^{\dagger}\right)}{\longrightarrow} H^{0}\left(\mathcal{F}_{0}^{1}\right)\right)=0$ and so we have $\mathbf{H}^{2}\left(\mathcal{F}^{\bullet}\right)=0$. In particular, we have $\omega\left(E, \nabla,\left\{l_{j}^{(i)}\right\}\right)=0$. Thus $\left(E, \nabla,\left\{l_{j}^{(i)}\right\}\right)$ can be lifted to a flat family $\left(\tilde{E}, \tilde{\nabla},\left\{\tilde{l}_{j}^{(i)}\right\}\right)$ over $A$ such that $\left(\tilde{E}, \tilde{\nabla},\left\{\tilde{l}_{j}^{(i)}\right\}\right) \otimes A / I \cong\left(E, \nabla,\left\{l_{j}^{(i)}\right\}\right)$ and that $\operatorname{det}\left(\tilde{E}, \tilde{\nabla},\left\{\tilde{l}_{j}^{(i)}\right\}\right)=\left(\left(L, \nabla_{L}\right), \boldsymbol{\nu}\right)$. Hence det is a smooth morphism.

THEOREM 1.3. For any $(x, \boldsymbol{\nu}) \in T \times N\left(d,\left(r_{j}^{(i)}\right)\right)$, the fiber $M_{\mathcal{C} / T}^{\boldsymbol{\alpha}}\left(d,\left(r_{j}^{(i)}\right)\right)_{(x, \boldsymbol{\nu})}:=$ $\pi^{-1}(x, \nu)$ is of equidimension 
$2 r^{2}(g-1)+2+2 \sum_{i=1}^{n} \sum_{j=0}^{s_{i}-1} \sum_{j^{\prime}>j} r_{j}^{(i)} r_{j^{\prime}}^{(i)}=2 r^{2}(g-1)+2+n r(r-1)-\sum_{i=1}^{n} \sum_{j=0}^{s_{i}-1} r_{j}^{(i)}\left(r_{j}^{(i)}-1\right)$ if $M_{\mathcal{C} / T}^{\boldsymbol{\alpha}}\left(d,\left(r_{j}^{(i)}\right)\right)_{(x, \boldsymbol{\nu})} \neq \emptyset$.

Proof. Since $M_{\mathcal{C} / T}^{\boldsymbol{\alpha}}\left(d,\left(r_{j}^{(i)}\right)\right)_{(x, \boldsymbol{\nu})}$ is smooth, it is sufficient to show that the tangent space $\Theta_{M_{\mathcal{C} / T}^{\alpha}\left(d,\left(r_{j}^{(i)}\right)\right)_{(x, \boldsymbol{\nu})}}(y)$ of $M_{\mathcal{C} / T}^{\alpha}\left(d,\left(r_{j}^{(i)}\right)\right)_{(x, \boldsymbol{\nu})}$ at any point $y=\left(E, \nabla,\left\{l_{j}^{(i)}\right\}\right) \in$ $M_{\mathcal{C} / T}^{\boldsymbol{\alpha}}\left(d,\left(r_{j}^{(i)}\right)\right)_{(x, \boldsymbol{\nu})}$ is of dimension

$$
2 r^{2}(g-1)+2+2 \sum_{i=1}^{n} \sum_{j=1}^{s_{i}-1} \sum_{j^{\prime}>j} r_{j}^{(i)} r_{j^{\prime}}^{(i)}
$$

Set

$$
\begin{aligned}
& \mathcal{F}^{0}:=\left\{a \in \mathcal{E} n d(E)|a|_{\left(\tilde{t}_{i}\right)_{x}}\left(l_{j}^{(i)}\right) \subset l_{j}^{(i)} \text { for any } i, j\right\} \\
& \mathcal{F}^{1}:=\left\{b \in \mathcal{E} n d(E) \otimes \Omega_{\mathcal{C} / T}^{1}\left(\tilde{t}_{1}+\cdots+\tilde{t}_{n}\right) \mid \operatorname{res}_{\left(\tilde{t}_{i}\right)_{x}}(b)\left(l_{j}^{(i)}\right) \subset l_{j+1}^{(i)} \text { for any } i, j\right\} \\
& \nabla^{\dagger}: \mathcal{F}^{0} \ni a \mapsto \nabla \circ a-a \circ \nabla \in \mathcal{F}^{1} .
\end{aligned}
$$

Note that we have an isomorphism

$$
\Theta_{M_{\mathcal{C} / T}^{\alpha}\left(d,\left(r_{j}^{(i)}\right)\right) / T \times N\left(d,\left(r_{j}^{(i)}\right)\right)}(y) \cong \mathbf{H}^{1}\left(\mathcal{F}^{\bullet}\right),
$$

where $\Theta_{M_{\mathcal{C} / T}^{\alpha}\left(d,\left(r_{j}^{(i)}\right)\right) / T \times N\left(d,\left(r_{j}^{(i)}\right)\right)}$ is the algebraic relative tangent bundle of $M_{\mathcal{C} / T}^{\boldsymbol{\alpha}}\left(d,\left(r_{j}^{(i)}\right)\right)$ over $T \times N\left(d,\left(r_{j}^{(i)}\right)\right)$. From the spectral sequence $H^{q}\left(\mathcal{F}^{p}\right) \Rightarrow \mathbf{H}^{p+q}\left(\mathcal{F}^{\bullet}\right)$, we obtain an exact sequence

$$
0 \longrightarrow \mathbb{C} \longrightarrow H^{0}\left(\mathcal{F}^{0}\right) \longrightarrow H^{0}\left(\mathcal{F}^{1}\right) \longrightarrow \mathbf{H}^{1}\left(\mathcal{F}^{\bullet}\right) \longrightarrow H^{1}\left(\mathcal{F}^{0}\right) \longrightarrow H^{1}\left(\mathcal{F}^{1}\right) \longrightarrow \mathbb{C} \longrightarrow 0 .
$$

So we have

$$
\begin{aligned}
\operatorname{dim} \mathbf{H}^{1}\left(\mathcal{F}^{\bullet}\right)= & \operatorname{dim} H^{0}\left(\mathcal{F}^{1}\right)+\operatorname{dim} H^{1}\left(\mathcal{F}^{0}\right)-\operatorname{dim} H^{0}\left(\mathcal{F}^{0}\right)-\operatorname{dim} H^{1}\left(\mathcal{F}^{1}\right)+2 \operatorname{dim} \mathbb{C} \mathbb{C} \\
= & \operatorname{dim} H^{0}\left(\left(\mathcal{F}^{0}\right)^{\vee} \otimes \Omega_{\mathcal{C} / T}^{1}\right)+\operatorname{dim} H^{1}\left(\mathcal{F}^{0}\right)-\operatorname{dim} H^{0}\left(\mathcal{F}^{0}\right) \\
& -\operatorname{dim} H^{1}\left(\left(\mathcal{F}^{0}\right)^{\vee} \otimes \Omega_{\mathcal{C} / T}^{1}\right)+2 \\
= & \operatorname{dim} H^{1}\left(\mathcal{F}^{0}\right)^{\vee}+\operatorname{dim} H^{1}\left(\mathcal{F}^{0}\right)-\operatorname{dim} H^{0}\left(\mathcal{F}^{0}\right)-\operatorname{dim} H^{0}\left(\mathcal{F}^{0}\right)^{\vee}+2 \\
= & 2-2 \chi\left(\mathcal{F}^{0}\right) .
\end{aligned}
$$

Here we used the isomorphism $\mathcal{F}^{1} \cong\left(\mathcal{F}^{0}\right)^{\vee} \otimes \Omega_{\mathcal{C} / T}^{1}$ and Serre duality. We define a subsheaf $\mathcal{E}_{1} \subset \mathcal{E} n d(E)$ by the exact sequence

$$
0 \longrightarrow \mathcal{E}_{1} \longrightarrow \mathcal{E} n d(E) \longrightarrow \bigoplus_{i=1}^{n} \operatorname{Hom}\left(l_{1}^{(i)}, l_{0}^{(i)} / l_{1}^{(i)}\right) \longrightarrow 0
$$


Inductively we define a subsheaf $\mathcal{E}_{k} \subset \mathcal{E} n d(E)$ by the exact sequence

$$
0 \longrightarrow \mathcal{E}_{k} \longrightarrow \mathcal{E}_{k-1} \longrightarrow \bigoplus_{i=1}^{n} \operatorname{Hom}\left(l_{k}^{(i)}, l_{k-1}^{(i)} / l_{k}^{(i)}\right) \longrightarrow 0 .
$$

Then we have $\mathcal{E}_{\max _{i}\left\{s_{i}-1\right\}}=\mathcal{F}^{0}$ and

$$
\begin{aligned}
\chi\left(\mathcal{F}^{0}\right) & =\chi(\mathcal{E} n d(E))-\sum_{i=1}^{n} \sum_{j=1}^{s_{i}-1} \operatorname{dim} \operatorname{Hom}\left(l_{j}^{(i)}, l_{j-1}^{(i)} / l_{j}^{(i)}\right) \\
& =r^{2}(1-g)-\sum_{i=1}^{n} \sum_{j=1}^{s_{i}-1} \sum_{j^{\prime}>j-1} r_{j-1}^{(i)} r_{j^{\prime}}^{(i)}
\end{aligned}
$$

So we have

$$
\operatorname{dim} \mathbf{H}^{1}\left(\mathcal{F}^{\bullet}\right)=2-\chi\left(\mathcal{F}^{0}\right)=2 r^{2}(g-1)+2+2 \sum_{i=1}^{n} \sum_{j=0}^{s_{i}-1} \sum_{j^{\prime}>j} r_{j}^{(i)} r_{j^{\prime}}^{(i)}
$$

\section{Riemann-Hilbert correspondence.}

Let $T, \mathcal{C}$ and $\tilde{\boldsymbol{t}}=\left(\tilde{t}_{1}, \ldots, \tilde{t}_{n}\right)$ be as in Section 1. Take a point $x \in T$. Then $\mathcal{C}_{x}$ is a smooth projective curve of genus $g$ over $\mathbb{C}$ and $\left(\tilde{t}_{1}\right)_{x}, \ldots,\left(\tilde{t}_{n}\right)_{x}$ are distinct points of $\mathcal{C}_{x}$. Consider the categorical quotient

$$
\operatorname{RP}_{r}\left(\mathcal{C}_{x}, \tilde{\boldsymbol{t}}_{x}\right):=\operatorname{Hom}\left(\pi_{1}\left(\mathcal{C}_{x} \backslash\left\{\left(\tilde{t}_{1}\right)_{x}, \ldots,\left(\tilde{t}_{n}\right)_{x}\right\}, *\right), G L_{r}(\mathbb{C})\right) / / G L_{r}(\mathbb{C})
$$

by the adjoint action. We set

$$
B:=\left\{\boldsymbol{b}:=\left(b_{j}^{(i)}\right)_{\substack{1 \leq i \leq n \\ 0 \leq j \leq s_{i}-1}} \mid b_{j}^{(i)} \in \mathbb{C} \text { for each } i, j \text { and } \prod_{i=1}^{n} \prod_{j=0}^{s_{i}-1}\left(b_{j}^{(i)}\right)^{r_{j}^{(i)}}=1\right\} .
$$

For $\boldsymbol{b} \in B$ and $x \in T$, we denote by $\mathrm{RP}_{r}\left(\mathcal{C}_{x}, \tilde{\boldsymbol{t}}_{x}, \boldsymbol{b}\right)$ the categorical quotient of

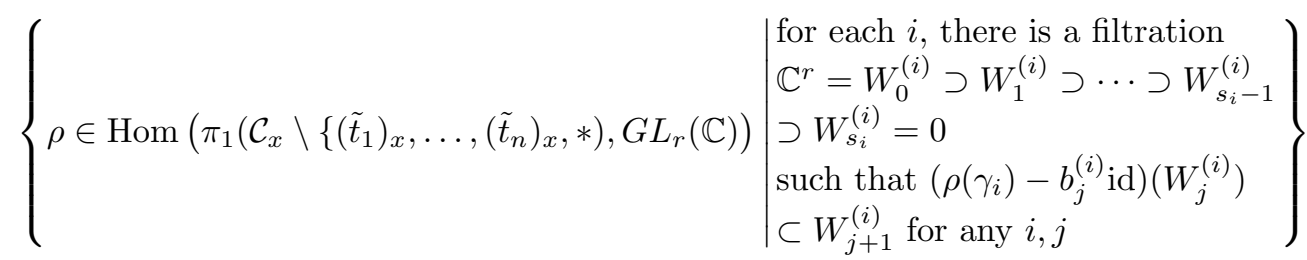

by the adjoint action of $G L_{r}(\mathbb{C})$, where $\gamma_{i}$ is a loop around $\left(\tilde{t}_{i}\right)_{x}$. Then we have a canonical closed immersion

$$
\mathrm{RP}_{r}\left(\mathcal{C}_{x}, \tilde{\boldsymbol{t}}_{x}, \boldsymbol{b}\right) \hookrightarrow \mathrm{RP}_{r}\left(\mathcal{C}_{x}, \tilde{\boldsymbol{t}}_{x}\right)
$$


For $\boldsymbol{\nu} \in N\left(d,\left(r_{j}^{(i)}\right)\right)$, consider the moduli space $M_{\mathcal{C} / T}^{\boldsymbol{\alpha}}\left(d,\left(r_{j}^{(i)}\right)\right)_{(x, \boldsymbol{\nu})}$. We define $\boldsymbol{b}=$ $\left(b_{j}^{(i)}\right)=r h(\boldsymbol{\nu})$ by

$$
b_{j}^{(i)}=\exp \left(-2 \pi \sqrt{-1} \nu_{j}^{(i)}\right)
$$

for any $i, j$. For $\left(E, \nabla,\left\{l_{j}^{(i)}\right\}\right) \in M_{\mathcal{C} / T}^{\boldsymbol{\alpha}}\left(d,\left(r_{j}^{(i)}\right)\right)_{(x, \nu)}$, $\left.\operatorname{ker} \nabla^{a n}\right|_{\mathcal{C}_{x} \backslash\left\{\left(\tilde{t}_{1}\right)_{x}, \ldots,\left(\tilde{t}_{n}\right)_{x}\right\}}$ becomes a local system and corresponds to a representation $\rho: \pi_{1}\left(\mathcal{C}_{x} \backslash\left\{\left(\tilde{t}_{1}\right)_{x}, \ldots,\left(\tilde{t}_{n}\right)_{x}\right\}, *\right) \rightarrow$ $G L_{r}(\mathbb{C})$. Then we put $\mathbf{R H}\left(E, \nabla,\left\{l_{j}^{(i)}\right\}\right):=[\rho] \in \operatorname{RP}_{r}\left(\mathcal{C}_{x}, \tilde{\boldsymbol{t}}_{x}, \boldsymbol{b}\right)$. So we can define a morphism

$$
\mathbf{R H}: M_{\mathcal{C} / T}^{\boldsymbol{\alpha}}\left(d,\left(r_{j}^{(i)}\right)\right)_{(x, \boldsymbol{\nu})} \longrightarrow \mathrm{RP}_{r}\left(\mathcal{C}_{x}, \tilde{\boldsymbol{t}}_{x}, \boldsymbol{b}\right) .
$$

Consider the scheme

$$
p: \tilde{M}_{\mathcal{C} / T}^{\boldsymbol{\alpha}}\left(d,\left(r_{j}^{(i)}\right)\right) \longrightarrow M_{\mathcal{C} / T}^{\boldsymbol{\alpha}}\left(d,\left(r_{j}^{(i)}\right)\right)
$$

such that for an affine scheme $U$ over $\mathcal{M}_{\mathcal{C} / T}^{\boldsymbol{\alpha}}\left(d,\left(r_{j}^{(i)}\right)\right)$,

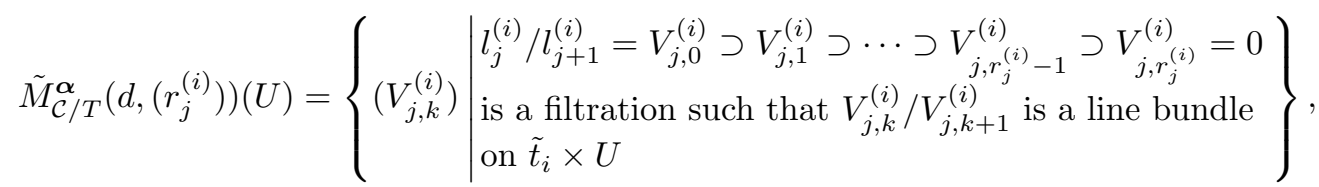

where $\mathcal{M}_{\mathcal{C} / T}^{\boldsymbol{\alpha}}\left(d,\left(r_{j}^{(i)}\right)\right)$ is the moduli functor of $\boldsymbol{\alpha}$-stable regular singular parabolic connections of spectral type $\left(r_{j}^{(i)}\right)$ and $\left(E, \nabla,\left\{l_{j}^{(i)}\right\}\right)$ is the member corresponding to $U \rightarrow \mathcal{M}_{\mathcal{C} / T}^{\boldsymbol{\alpha}}\left(d,\left(r_{j}^{(i)}\right)\right)$. Then $\tilde{M}_{\mathcal{C} / T}^{\boldsymbol{\alpha}}\left(d,\left(r_{j}^{(i)}\right)\right)$ is a flag scheme over $M_{\mathcal{C} / T}^{\boldsymbol{\alpha}}\left(d,\left(r_{j}^{(i)}\right)\right)$ and so $p$ is a smooth projective surjective morphism. A point of $\tilde{M}_{\mathcal{C} / T}^{\alpha}\left(d,\left(r_{j}^{(i)}\right)\right)$ corresponds to a regular singular parabolic connection considered in [4]. Assume that we can choose $\boldsymbol{\alpha}$ so that $\boldsymbol{\alpha}$-stable $\Leftrightarrow \boldsymbol{\alpha}$-semistable. If we choose $\boldsymbol{\alpha}^{\prime}=\left(\left(\alpha^{\prime}\right)_{k}^{(i)}\right)_{1<k<r}^{\substack{1 \leq i \leq n \\ 1<k<r}}$ suitably, any parabolic connection $\left(E, \nabla,\left\{l_{j}^{(i)}\right\},\left\{V_{j, k}^{(i)}\right\}\right)$ in $\tilde{M}_{\mathcal{C} / T}^{\boldsymbol{\alpha}}\left(d,\left(r_{j}^{(i)}\right)\right)$ is automatically $\boldsymbol{\alpha}^{\prime}$-stable. So we can define an inclusion

$$
\iota: \tilde{M}_{\mathcal{C} / T}^{\boldsymbol{\alpha}}\left(d,\left(r_{j}^{(i)}\right)\right) \hookrightarrow M_{\mathcal{C} / T}^{\boldsymbol{\alpha}^{\prime}}(\tilde{\boldsymbol{t}}, r, d)
$$

where $M_{\mathcal{C} / T}^{\boldsymbol{\alpha}^{\prime}}(\tilde{\boldsymbol{t}}, r, d)$ is the moduli space of $\boldsymbol{\alpha}^{\prime}$-stable regular singular parabolic connections defined in [4, Theorem 2.1]. If we take $\boldsymbol{\alpha}^{\prime}$ suitably, $\iota$ becomes a closed immersion.

For $\boldsymbol{\nu}=\left(\nu_{j}^{(i)}\right) \in N\left(d,\left(r_{j}^{(i)}\right)\right)$, we define $\boldsymbol{\nu}^{\prime}=\left(\left(\nu^{\prime}\right)_{q}^{(i)}\right)_{0 \leq i \leq r-1}^{\substack{1 \leq i \leq n \\ 0 \leq 4}}$ by $\left(\nu^{\prime}\right)_{q}^{(i)}=\nu_{j}^{(i)}$ if $q=m+\sum_{j^{\prime}<j} r_{j^{\prime}}^{(i)}$ with $0 \leq m \leq r_{j}^{(i)}-1$. Now assume that $r n-2 r-2>0$ if $g=0$, $n>1$ if $g=1$ and $n \geq 1$ if $g=2$. Since the Riemann-Hilbert morphism

$$
\mathbf{R H}: M_{\mathcal{C} / T}^{\boldsymbol{\alpha}^{\prime}}(\tilde{\boldsymbol{t}}, r, d)_{\left(x, \boldsymbol{\nu}^{\prime}\right)} \longrightarrow R P_{r}(\mathcal{C}, \tilde{\boldsymbol{t}})_{r h\left(\boldsymbol{\nu}^{\prime}\right)}
$$

is a proper surjective morphism by [4], the restriction 


$$
\left.\mathbf{R H}\right|_{\tilde{M}_{\mathcal{C} / T}^{\alpha}\left(d,\left(r_{j}^{(i)}\right)\right)_{(x, \boldsymbol{\nu})}}: \tilde{M}_{\mathcal{C} / T}^{\boldsymbol{\alpha}}\left(d,\left(r_{j}^{(i)}\right)\right)_{(x, \boldsymbol{\nu})} \longrightarrow \mathrm{RP}_{r}\left(\mathcal{C}_{x}, \tilde{\boldsymbol{t}}_{x}, \boldsymbol{b}\right)
$$

is also proper. We have a commutative diagram

$$
\begin{aligned}
& \tilde{M}_{\mathcal{C} / T}^{\boldsymbol{\alpha}}\left(d,\left(r_{j}^{(i)}\right)\right)_{(x, \boldsymbol{\nu})} \quad \stackrel{p}{\longrightarrow} \quad M_{\mathcal{C} / T}^{\boldsymbol{\alpha}}\left(d,\left(r_{j}^{(i)}\right)\right)_{(x, \boldsymbol{\nu})} \\
& \left.\mathbf{R H}\right|_{\tilde{M}_{\mathcal{C} / T}^{\alpha}\left(d,\left(r_{j}^{(i)}\right)\right)_{(x, \boldsymbol{\nu})}} \searrow \operatorname{RP}_{r}\left(\mathcal{C}_{x}, \tilde{\boldsymbol{t}}_{x}, \boldsymbol{b}\right) .
\end{aligned}
$$

Since $p$ is surjective, the morphism

$$
\mathbf{R H}: M_{\mathcal{C} / T}^{\boldsymbol{\alpha}}\left(d,\left(r_{j}^{(i)}\right)\right)_{(x, \boldsymbol{\nu})} \longrightarrow \mathrm{RP}_{r}\left(\mathcal{C}_{x}, \tilde{\boldsymbol{t}}_{x}, \boldsymbol{b}\right)
$$

becomes a proper morphism.

REMARK 2.1. Yamakawa gives in [13, 4.3, 4.4], the Riemann-Hilbert isomorphism from the moduli space $M_{\mathcal{C} / T}^{\alpha}\left(d,\left(r_{j}^{(i)}\right)\right)_{(x, \nu)}$ to the moduli space of stable filtered local systems which is constructed as a quiver variety. The properness of the morphism $\mathbf{R H}: M_{\mathcal{C} / T}^{\boldsymbol{\alpha}}\left(d,\left(r_{j}^{(i)}\right)\right)_{(x, \boldsymbol{\nu})} \longrightarrow \mathrm{RP}_{r}\left(\mathcal{C}_{x}, \tilde{\boldsymbol{t}}_{x}, \boldsymbol{b}\right)$ can be obtained also from this Yamakawa's precise result.

REMARK 2.2. It is somewhat a complicated problem whether the morphism RH : $M_{\mathcal{C} / T}^{\boldsymbol{\alpha}}\left(d,\left(r_{j}^{(i)}\right)\right)_{(x, \boldsymbol{\nu})} \rightarrow \mathrm{RP}_{r}\left(\mathcal{C}_{x}, \tilde{\boldsymbol{t}}_{x}, \boldsymbol{b}\right)$ defined above is surjective. For example, it happens that for $g=0$ and for small $n$, the moduli space $M_{\mathcal{C} / T}^{\boldsymbol{\alpha}}\left(d,\left(r_{j}^{(i)}\right)\right)_{(x, \boldsymbol{\nu})}$ becomes empty but the moduli space $\mathrm{RP}_{r}\left(\mathcal{C}_{x}, \tilde{\boldsymbol{t}}_{x}, \boldsymbol{b}\right)$ is not empty.

\section{Relative symplectic form on the moduli space.}

TheOrem 3.1. Assume that we can take $\boldsymbol{\alpha}$ so that $\boldsymbol{\alpha}$-stable $\Leftrightarrow \boldsymbol{\alpha}$-semistable. Then there exists a relative symplectic form

$$
\omega \in H^{0}\left(M_{\mathcal{C} / T}^{\alpha}\left(d,\left(r_{j}^{(i)}\right)\right), \Omega_{M_{\mathcal{C} / T}^{\alpha}\left(d,\left(r_{j}^{(i)}\right)\right) / T \times N\left(d,\left(r_{j}^{(i)}\right)\right)}^{2}\right) .
$$

REMARK 3.1. We need some assumption on $\left(r_{j}^{(i)}\right)$ for the existence of such $\boldsymbol{\alpha}$. For example, if some $r_{j}^{(i)}$ is coprime to $r$, then we can take such $\boldsymbol{\alpha}$.

Proof. There are an affine scheme $U$ and an étale surjective morphism $\tau: U \rightarrow$ $M_{\mathcal{C} / T}^{\alpha}\left(d,\left(r_{j}^{(i)}\right)\right)$, which factors through the moduli functor $\mathcal{M}_{\mathcal{C} / T}^{\boldsymbol{\alpha}}\left(d,\left(r_{j}^{(i)}\right)\right)$, namely there is a universal family $\left(\tilde{E}, \tilde{\nabla},\left\{\tilde{l}_{j}^{(i)}\right\}\right)$ on $\mathcal{C} \times_{T} U$. We define a complex $\mathcal{F}^{\bullet}$ on $\mathcal{C} \times_{T} U$ by

$$
\begin{aligned}
& \mathcal{F}^{0}:=\left\{a \in \mathcal{E} n d(\tilde{E})|a|_{\left(\tilde{t}_{i}\right)_{U}}\left(\tilde{l}_{j}^{(i)}\right) \subset \tilde{l}_{j}^{(i)} \text { for any } i, j\right\} \\
& \mathcal{F}^{1}:=\left\{b \in \mathcal{E} n d(\tilde{E}) \otimes \Omega_{\mathcal{C} / T}^{1}\left(\tilde{t}_{1}+\cdots+\tilde{t}_{n}\right) \mid \operatorname{res}_{\left(\tilde{t}_{i}\right)_{U}}(b)\left(\tilde{l}_{j}^{(i)}\right) \subset \tilde{l}_{j+1}^{(i)} \text { for any } i, j\right\} \\
& \nabla^{\dagger}: \mathcal{F}^{0} \ni a \mapsto \tilde{\nabla} \circ a-a \circ \tilde{\nabla} \in \mathcal{F}^{1} .
\end{aligned}
$$


Let $\pi_{U}: \mathcal{C} \times_{T} U \rightarrow U$ be the projection. Then we have

$$
\Theta_{U / T \times N\left(d,\left(r_{j}^{(i)}\right)\right)} \cong \tau^{*}\left(\Theta_{M_{\mathcal{C} / T}^{\alpha}\left(d,\left(r_{j}^{(i)}\right)\right) / T \times N\left(d,\left(r_{j}^{(i)}\right)\right)}\right) \cong \mathbf{R}^{1}\left(\pi_{U}\right)_{*}\left(\mathcal{F}^{\bullet}\right) .
$$

Take an affine open covering $\mathcal{C} \times_{T} U=\bigcup_{\alpha} U_{\alpha}$ and a member $v \in H^{0}\left(U, \mathbf{R}^{1}\left(\pi_{U}\right)_{*}\left(\mathcal{F}^{\bullet}\right)\right)=$ $\mathbf{H}^{1}\left(\mathcal{C} \times_{T} U, \mathcal{F}^{\bullet}\right) . \quad v$ is given by $\left[\left(\left\{u_{\alpha \beta}\right\},\left\{v_{\alpha}\right\}\right)\right]$, where $\left\{u_{\alpha \beta}\right\} \in C^{1}\left(\left\{U_{\alpha}\right\}, \mathcal{F}^{0}\right),\left\{v_{\alpha}\right\} \in$ $C^{0}\left(\left\{U_{\alpha}\right\}, \mathcal{F}^{1}\right)$ and

$$
d\left\{u_{\alpha \beta}\right\}=\left\{u_{\beta \gamma}-u_{\alpha \gamma}+u_{\alpha \beta}\right\}=0, \quad \nabla^{\dagger}\left(\left\{u_{\alpha \beta}\right\}\right)=\left\{v_{\beta}-v_{\alpha}\right\}=d\left\{v_{\alpha}\right\} .
$$

We define a pairing

$$
\omega_{U}: \mathbf{H}^{1}\left(\mathcal{C} \times_{T} U, \mathcal{F}^{\bullet}\right) \times \mathbf{H}^{1}\left(\mathcal{C} \times_{T} U, \mathcal{F}^{\bullet}\right) \longrightarrow \mathbf{H}^{2}\left(\mathcal{C} \times_{T} U, \Omega_{\mathcal{C} \times_{T} U / U}\right) \cong H^{0}\left(U, \mathcal{O}_{U}\right)
$$

by

$\omega_{U}\left(\left[\left(\left\{u_{\alpha \beta}\right\},\left\{v_{\alpha}\right\}\right)\right],\left[\left(\left\{u_{\alpha \beta}^{\prime}\right\},\left\{v_{\alpha}^{\prime}\right\}\right)\right]\right):=\left[\left(\left\{\operatorname{Tr}\left(u_{\alpha \beta} \circ u_{\beta \gamma}^{\prime}\right)\right\},-\left\{\operatorname{Tr}\left(u_{\alpha \beta} \circ v_{\beta}^{\prime}\right)-\operatorname{Tr}\left(v_{\alpha} \circ u_{\alpha \beta}^{\prime}\right)\right\}\right)\right]$.

By definition, we can easily see that $\omega_{U}$ descends to a pairing

$$
\omega: \Theta_{M_{\mathcal{C} / T}^{\alpha}\left(d,\left(r_{j}^{(i)}\right)\right) / T \times N\left(d,\left(r_{j}^{(i)}\right)\right)} \times \Theta_{M_{\mathcal{C} / T}^{\alpha}\left(d,\left(r_{j}^{(i)}\right)\right) / T \times N\left(d,\left(r_{j}^{(i)}\right)\right)} \longrightarrow \mathcal{O}_{M_{\mathcal{C} / T}^{\alpha}\left(d,\left(r_{j}^{(i)}\right)\right)}
$$

Take any $\mathbb{C}$-valued point $y=\left(E, \nabla,\left\{l_{j}^{(i)}\right\}\right) \in M_{\mathcal{C} / T}^{\boldsymbol{\alpha}}\left(d,\left(r_{j}^{(i)}\right)\right)(\mathbb{C})$ over $(x, \boldsymbol{\nu}) \in T \times$ $N\left(d,\left(r_{j}^{(i)}\right)\right)$. Then a tangent vector $v \in \Theta_{M_{\mathcal{C} / T}^{\alpha}\left(d,\left(r_{j}^{(i)}\right)\right) / T \times N\left(d,\left(r_{j}^{(i)}\right)\right)}(y)$ corresponds to a $\mathbb{C}[t] /\left(t^{2}\right)$-valued point $\left(E^{v}, \nabla^{v},\left\{\left(l^{v}\right)_{j}^{(i)}\right\}\right) \in \mathcal{M}_{\mathcal{C} / T}^{\boldsymbol{\alpha}}\left(d,\left(r_{j}^{(i)}\right)\right)_{(x, \boldsymbol{\nu})}\left(\mathbb{C}[t] /\left(t^{2}\right)\right)$ such that $\left(E^{v}, \nabla^{v},\left\{\left(l^{v}\right)_{j}^{(i)}\right\}\right) \otimes \mathbb{C}[t] /(t) \cong\left(E, \nabla,\left\{l_{j}^{(i)}\right\}\right)$. We can check that $\omega(v, v)$ is nothing but the obstruction class for the lifting of $\left(E^{v}, \nabla^{v},\left\{\left(l^{v}\right)_{j}^{(i)}\right\}\right)$ to a member of

$$
\mathcal{M}_{\mathcal{C} / T}^{\boldsymbol{\alpha}}\left(d,\left(r_{j}^{(i)}\right)\right)_{(x, \boldsymbol{\nu})}\left(\mathbb{C}[t] /\left(t^{3}\right)\right)
$$

Since $M_{\mathcal{C} / T}^{\boldsymbol{\alpha}}\left(d,\left(r_{j}^{(i)}\right)\right)_{(x, \boldsymbol{\nu})}$ is smooth, we have $\omega(v, v)=0$. Thus $\omega$ is skew symmetric.

Let

$$
\xi: \Theta_{M_{\mathcal{C} / T}^{\alpha}\left(d,\left(r_{j}^{(i)}\right)\right) / T \times N\left(d,\left(r_{j}^{(i)}\right)\right)} \longrightarrow \Theta_{M_{\mathcal{C} / T}^{\alpha}\left(d,\left(r_{j}^{(i)}\right)\right) / T \times N\left(d,\left(r_{j}^{(i)}\right)\right)}^{\vee}
$$

be the homomorphism induced by $\omega$. For any $\mathbb{C}$-valued point $y \in M_{\mathcal{C} / T}^{\alpha}\left(d,\left(r_{j}^{(i)}\right)\right)(\mathbb{C})$

$$
\begin{aligned}
\xi(y): \mathbf{H}^{1}\left(\mathcal{F}^{\bullet}(y)\right)=\Theta_{M_{\mathcal{C} / T}^{\alpha}\left(d,\left(r_{j}^{(i)}\right)\right) / T \times N\left(d,\left(r_{j}^{(i)}\right)\right)}(y) \longrightarrow & \Theta_{M_{\mathcal{C} / T}^{\boldsymbol{\alpha}}\left(d,\left(r_{j}^{(i)}\right)\right) / T \times N\left(d,\left(r_{j}^{(i)}\right)\right)}^{\vee}(y) \\
& =\mathbf{H}^{1}\left(\mathcal{F}^{\bullet}(y)\right)^{\vee}
\end{aligned}
$$

induces an exact commutative diagram 


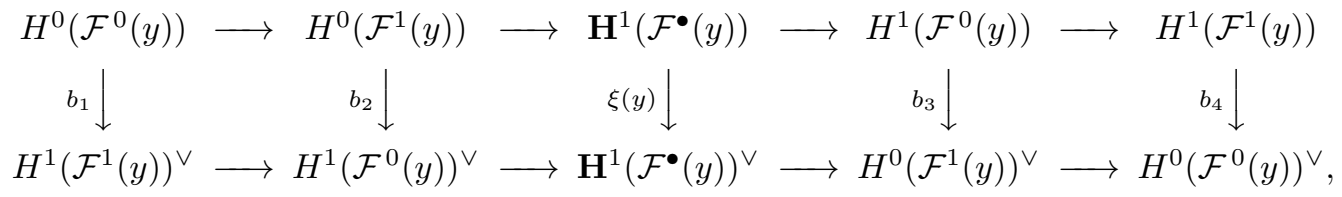

where $b_{1}, b_{2}, b_{3}, b_{4}$ are isomorphisms induced by $\mathcal{F}^{0}(y) \cong \mathcal{F}^{1}(y)^{\vee} \otimes \Omega_{\mathcal{C}_{y}}^{1}, \mathcal{F}^{1}(y) \cong$ $\mathcal{F}^{0}(y)^{\vee} \otimes \Omega_{\mathcal{C}_{y}}^{1}$ and Serre duality. Thus $\xi(y)$ becomes an isomorphism by the five lemma.

Now we will prove that $\omega$ is $d$-closed. As is explained in Section 2, we have a smooth projective surjective morphism $p: \tilde{M}_{\mathcal{C} / T}^{\alpha}\left(d,\left(r_{j}^{(i)}\right)\right)_{(x, \boldsymbol{\nu})} \rightarrow M_{\mathcal{C} / T}^{\boldsymbol{\alpha}}\left(d,\left(r_{j}^{(i)}\right)\right)_{(x, \boldsymbol{\nu})}$ and a closed immersion $\iota: \tilde{M}_{\mathcal{C} / T}^{\boldsymbol{\alpha}}\left(d,\left(r_{j}^{(i)}\right)\right)_{(x, \boldsymbol{\nu})} \hookrightarrow M_{\mathcal{C} / T}^{\boldsymbol{\alpha}^{\prime}}(\tilde{\boldsymbol{t}}, r, d)_{\left(x, \boldsymbol{\nu}^{\prime}\right)}$. Take any closed point $y \in M_{\mathcal{C} / T}^{\boldsymbol{\alpha}}\left(d,\left(r_{j}^{(i)}\right)\right)_{(x, \boldsymbol{\nu})}$. Then there is a subscheme $U \subset \tilde{M}_{\mathcal{C} / T}^{\boldsymbol{\alpha}}\left(d,\left(r_{j}^{(i)}\right)\right)_{(x, \boldsymbol{\nu})}$ such that $\left.p\right|_{U}: U \rightarrow M_{\mathcal{C} / T}^{\boldsymbol{\alpha}}\left(d,\left(r_{j}^{(i)}\right)\right)_{(x, \boldsymbol{\nu})}$ is étale and $y \in p(U)$. We can take a closed point $y^{\prime} \in U$ such that $p\left(y^{\prime}\right)=y$. Then $y$ corresponds to a member $\left(E, \nabla,\left\{l_{j}^{(i)}\right\}\right) \in$ $M_{\mathcal{C} / T}^{\boldsymbol{\alpha}}\left(d,\left(r_{j}^{(i)}\right)\right)_{(x, \boldsymbol{\nu})}$ and $y^{\prime}$ corresponds to a member $\left(E, \nabla,\left\{l_{j}^{(i)}\right\},\left\{V_{j, k}^{(i)}\right\}\right)$. Take tangent vectors $v, w \in \Theta_{U}\left(y^{\prime}\right)$. Since $\Theta_{U}\left(y^{\prime}\right) \cong \Theta_{M_{\mathcal{C} / T}^{\alpha}\left(d,\left(r_{j}^{(i)}\right)\right)_{(x, \nu)}}(y)$, we can regard $v, w$ as elements of $\mathbf{H}^{1}\left(\mathcal{F}^{\bullet}(y)\right)$. Put

$$
\begin{aligned}
& \tilde{\mathcal{F}}^{0}:=\left\{\begin{array}{l}
\left.a \in \mathcal{E} n d(E) \mid \begin{array}{l}
\left.a\right|_{\left(\tilde{t}_{i}\right)_{x}}\left(l_{j}^{(i)}\right) \subset l_{j}^{(i)} \text { for any } i, j \text { and } \\
\text { for the induced morphism } a_{j}^{(i)}: l_{j}^{(i)} / l_{j+1}^{(i)} \rightarrow l_{j}^{(i)} / l_{j+1}^{(i)} \\
\text { we have }\left(a_{j}^{(i)} \otimes \mathrm{id}\right)\left(V_{j, k}^{(i)}\right) \subset V_{j, k}^{(i)} \text { for any } i, j, k
\end{array}\right\}, \\
\tilde{\mathcal{F}}^{1}:=\left\{\begin{array}{l}
\operatorname{res}_{\left(\tilde{t}_{i}\right)_{x}}(b)\left(l_{j}^{(i)}\right) \subset l_{j}^{(i)} \text { for any } i, j \text { and } \\
\text { for the induced morphism } b_{j}^{(i)}: l_{j}^{(i)} / l_{j+1}^{(i)} \rightarrow l_{j}^{(i)} / l_{j+1}^{(i)} \\
\text { we have } b_{j}^{(i)}\left(V_{j, k}^{(i)}\right) \subset V_{j, k+1}^{(i)} \text { for any } i, j, k
\end{array}\right.
\end{array}\right\}, \\
& \tilde{\nabla}^{\dagger}: \tilde{\mathcal{F}}^{0} \ni a \mapsto \nabla \circ a-a \circ \nabla \in \tilde{\mathcal{F}}^{1} .
\end{aligned}
$$

We have a canonical commutative diagram

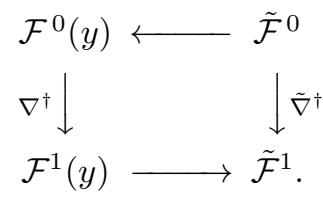

Then we have

$$
\begin{aligned}
& \Theta_{U}\left(y^{\prime}\right) \cong \mathbf{H}^{1}\left(\mathcal{F}^{\bullet}(y)\right), \\
& \Theta_{\tilde{M}_{\mathcal{C} / T}^{\alpha}\left(d,\left(r_{j}^{(i)}\right)\right)_{(x, \boldsymbol{\nu})}}\left(y^{\prime}\right) \cong \mathbf{H}^{1}\left(\tilde{\mathcal{F}}^{0} \rightarrow \mathcal{F}^{1}(y)\right) \text {, } \\
& \Theta_{M_{\mathcal{C} / T}^{\alpha^{\prime}}(\tilde{\boldsymbol{t}}, r, d)_{\left(x, \nu^{\prime}\right)}}\left(y^{\prime}\right) \cong \mathbf{H}^{1}\left(\tilde{\mathcal{F}}^{0} \rightarrow \tilde{\mathcal{F}}^{1}\right),
\end{aligned}
$$

and canonical homomorphisms

$$
\begin{aligned}
\Theta_{M_{\mathcal{C} / T}^{\alpha^{\prime}}(\tilde{\boldsymbol{t}}, r, d)_{\left(x, \boldsymbol{\nu}^{\prime}\right)}}\left(y^{\prime}\right) \cong \mathbf{H}^{1}\left(\tilde{\mathcal{F}}^{0} \rightarrow \tilde{\mathcal{F}}^{1}\right) \hookleftarrow \mathbf{H}^{1}\left(\tilde{\mathcal{F}}^{0} \rightarrow \mathcal{F}^{1}\left(y^{\prime}\right)\right) \stackrel{p_{*}}{\longrightarrow} & \mathbf{H}^{1}\left(\mathcal{F}^{\bullet}\left(y^{\prime}\right)\right) \\
& \cong \Theta_{M_{C}^{\alpha}\left(d,\left(r_{j}^{(i)}\right)\right)_{\nu}}(y) .
\end{aligned}
$$


There is a canonical symplectic form $\tilde{\omega}$ on $M_{\mathcal{C} / T}^{\boldsymbol{\alpha}^{\prime}}(\tilde{\boldsymbol{t}}, r, d)_{\left(x, \boldsymbol{\nu}^{\prime}\right)}$. There exists a splitting $s: \mathbf{H}^{1}\left(\mathcal{F}^{\bullet}\left(y^{\prime}\right)\right) \hookrightarrow \mathbf{H}^{1}\left(\tilde{F}^{0} \rightarrow \mathcal{F}^{1}\left(y^{\prime}\right)\right)$ of the surjection $p_{*}: \mathbf{H}^{1}\left(\tilde{F}^{0} \rightarrow \mathcal{F}^{1}\left(y^{\prime}\right)\right) \rightarrow$ $\mathbf{H}^{1}\left(\mathcal{F}^{\bullet}\left(y^{\prime}\right)\right)$ determined by $U$. Take an affine open covering $\mathcal{C}_{x}=\bigcup_{\alpha} U_{\alpha}$. The tangent vectors $v, w$ can be represented by $\left(\left\{a_{\alpha \beta}\right\},\left\{b_{\alpha}\right\}\right)$ and $\left(\left\{a_{\alpha \beta}^{\prime}\right\},\left\{b_{\alpha}^{\prime}\right\}\right)$, respectively, where $\left\{a_{\alpha \beta}\right\},\left\{a_{\alpha \beta}^{\prime}\right\} \in C^{1}\left(\left\{U_{\alpha}\right\}, \mathcal{F}^{0}\left(y^{\prime}\right)\right)$ and $\left\{b_{\alpha}\right\},\left\{b_{\alpha}^{\prime}\right\} \in C^{0}\left(\left\{U_{\alpha}\right\}, \mathcal{F}^{1}\left(y^{\prime}\right)\right)$. Replacing $a_{\alpha \beta}, a_{\alpha \beta}^{\prime}, b_{\alpha}, b_{\alpha}^{\prime}$, we may have that $s(v)$ and $s(w)$ can be represented by $\left(\left\{a_{\alpha \beta}\right\},\left\{b_{\alpha}\right\}\right)$ and $\left(\left\{a_{\alpha \beta}^{\prime}\right\},\left\{b_{\alpha}^{\prime}\right\}\right)$, respectively with $\left\{a_{\alpha \beta}\right\},\left\{a_{\alpha \beta}^{\prime}\right\} \in C^{1}\left(\left\{U_{\alpha}\right\}, \tilde{\mathcal{F}}^{0}\right)$. Then we have

$$
\tilde{\omega}\left(\iota_{*}(s(v)), \iota_{*}(s(w))\right)=\left[\left(\left\{\operatorname{Tr}\left(a_{\alpha \beta} \circ a_{\beta \gamma}^{\prime}\right)\right\},-\left\{\operatorname{Tr}\left(a_{\alpha \beta} \circ b_{\beta}^{\prime}\right)-\operatorname{Tr}\left(b_{\alpha} \circ a_{\alpha \beta}^{\prime}\right)\right\}\right)\right]=\omega(v, w),
$$

which means that $\left.\tilde{\omega}\right|_{U}=\left(\left.p\right|_{U}\right)^{*}(\omega)$. Since $\tilde{\omega}$ is $d$-closed by [4, Proposition 7.3], $\left(\left.p\right|_{U}\right)^{*}(\omega)$ is also $d$-closed. Thus $\omega$ is $d$-closed, because $\left.p\right|_{U}: U \rightarrow M_{\mathcal{C} / T}^{\alpha}\left(d,\left(r_{j}^{(i)}\right)\right)_{(x, \nu)}$ is étale.

\section{Isomonodromic deformation.}

Let $T$ be an algebraic scheme over $\mathbb{C}$, which is a smooth covering of the moduli stack of $n$-pointed smooth projective curves of genus $g$. Take a universal family $(\mathcal{C}, \tilde{\boldsymbol{t}})$ over $T$. For the spectral type $\left(r_{j}^{(i)}\right)$, assume that we can take a parabolic weight $\boldsymbol{\alpha}$ such that $\boldsymbol{\alpha}$-stable $\Leftrightarrow \boldsymbol{\alpha}$-semistable. We choose $\boldsymbol{\alpha}^{\prime}$ as in Section 2. As is stated in [4, Propostion 8.1], there is an algebraic splitting

$$
D: \pi^{*}\left(\Theta_{T}\right) \longrightarrow \Theta_{M_{\mathcal{C} / T}^{\boldsymbol{\alpha}^{\prime}}(\tilde{\boldsymbol{t}}, r, d)}
$$

of the canonical surjection $\pi_{*}: \Theta_{M_{\mathcal{C} / T}^{\alpha^{\prime}}(\tilde{\boldsymbol{t}}, r, d)} \rightarrow \pi^{*}\left(\Theta_{T}\right)$, where $\pi: M_{\mathcal{C} / T}^{\alpha^{\prime}}(\tilde{\boldsymbol{t}}, r, d) \rightarrow$ $T$ is the structure morphism. By the construction of $D$ in [4, Proposition 8.1], we can see that the image of $\left.D\right|_{\tilde{M}_{\mathcal{C} / T}^{\alpha}\left(d,\left(r_{j}^{(i)}\right)\right)}$ is contained in $\Theta_{\tilde{M}_{\mathcal{C} / T}^{\alpha}\left(d,\left(r_{j}^{(i)}\right)\right)} \subset$ $\left.\Theta_{M_{\mathcal{C} / T}^{\alpha^{\prime}}(\tilde{\boldsymbol{t}}, r, d)}\right|_{\tilde{M}_{\mathcal{C} / T}^{\alpha}\left(d,\left(r_{j}^{(i)}\right)\right)}$. Since $D\left(\pi^{*}\left(\Theta_{T}\right)\right) \subset \Theta_{M_{\mathcal{C} / T}^{\alpha^{\prime}(\tilde{\boldsymbol{t}}, r, d)}}$ satisfies the integrability condition, $\left.D\right|_{\tilde{M}_{\mathcal{C} / T}^{\alpha}\left(d,\left(r_{j}^{(i)}\right)\right)}\left(\left(\left.\pi\right|_{\tilde{M}_{\mathcal{C} / T}^{\alpha}\left(d,\left(r_{j}^{(i)}\right)\right)}\right) *\left(\Theta_{T}\right)\right) \subset \Theta_{\tilde{M}_{\mathcal{C} / T}^{\alpha}\left(d,\left(r_{j}^{(i)}\right)\right)}$ also satisfies the integrability condition. Consider the projective surjective morphism

$$
p: \tilde{M}_{\mathcal{C} / T}^{\boldsymbol{\alpha}}\left(d,\left(r_{j}^{(i)}\right)\right) \longrightarrow M_{\mathcal{C} / T}^{\boldsymbol{\alpha}}\left(d,\left(r_{j}^{(i)}\right)\right)
$$

as in Section 2. Note that the geometric fibers of $p$ are irreducible. Then we obtain a composition of homomorphisms

$$
\begin{aligned}
D^{\prime}:\left(\pi^{\prime}\right)^{*}\left(\Theta_{T}\right) \stackrel{\sim}{\longrightarrow} p_{*}\left(\pi^{*}\left(\Theta_{T}\right)\right) \stackrel{p_{*}\left(\left.D\right|_{\tilde{M}_{\mathcal{C} / T}^{\alpha}\left(d,\left(r_{j}^{(i)}\right)\right)}\right)}{\longrightarrow} p_{*}\left(\Theta_{\tilde{M}_{\mathcal{C} / T}^{\alpha}\left(d,\left(r_{j}^{(i)}\right)\right)}\right) \\
\longrightarrow p_{*}\left(p^{*}\left(\Theta_{M_{\mathcal{C} / T}^{\alpha}\left(d,\left(r_{j}^{(i)}\right)\right)}\right)\right) \stackrel{\sim}{\longrightarrow} \Theta_{M_{\mathcal{C} / T}^{\alpha}\left(d,\left(r_{j}^{(i)}\right)\right)}
\end{aligned}
$$

and $D^{\prime}$ is an algebraic splitting of the canonical surjective homomorphism $\pi_{*}^{\prime}: \Theta_{M_{\mathcal{C} / T}^{\alpha}\left(d,\left(r_{j}^{(i)}\right)\right)} \rightarrow\left(\pi^{\prime}\right)^{*}\left(\Theta_{T}\right)$, where $\pi^{\prime}: M_{\mathcal{C} / T}^{\alpha}\left(d,\left(r_{j}^{(i)}\right)\right) \rightarrow T$ is the structure morphism. Since $\left.D\right|_{\tilde{M}_{\mathcal{C} / T}^{\alpha}\left(d,\left(r_{j}^{(i)}\right)\right)}\left(\left(\left.\pi\right|_{\tilde{M}_{\mathcal{C} / T}^{\alpha}\left(d,\left(r_{j}^{(i)}\right)\right)}\right)^{*}\left(\Theta_{T}\right)\right) \subset \Theta_{\tilde{M}_{\mathcal{C} / T}^{\alpha}\left(d,\left(r_{j}^{(i)}\right)\right)}$ satisfies the integrability condition, $D^{\prime}\left(\left(\pi^{\prime}\right)^{*}\left(\Theta_{T}\right)\right) \subset \Theta_{M_{\mathcal{C} / T}^{\alpha}\left(d,\left(r_{j}^{(i)}\right)\right)}$ also satisfies the integrability con- 
dition. The corresponding foliation $\mathcal{F}_{M_{\mathcal{C} / T}^{\alpha}\left(d,\left(r_{j}^{(i)}\right)\right)}$ is nothing but the isomonodromic deformation.

TheOREM 4.1. Assume that $r n-2 r-2>0$ if $g=0, n>1$ if $g=1$ and $n>0$ if $g \geq 2$. Moreover, assume that we can take $\boldsymbol{\alpha}$ so that $\boldsymbol{\alpha}$-stable $\Leftrightarrow \boldsymbol{\alpha}$-semistable. Then the isomonodromic foliation $\mathcal{F}_{M_{\mathcal{C} / T}^{\alpha}\left(d,\left(r_{j}^{(i)}\right)\right)}$ determined by $D^{\prime}$ satisfies the geometric Painlevé property, namely for any path $\gamma:[0,1] \rightarrow T$ and for any point $x \in M_{\mathcal{C} / T}^{\alpha}\left(d,\left(r_{j}^{(i)}\right)\right)$ with $\pi^{\prime}(x)=\gamma(0)$, there is a unique path $\tilde{\gamma}:[0,1] \rightarrow M_{\mathcal{C} / T}^{\alpha}\left(d,\left(r_{j}^{(i)}\right)\right)$ which lies in a leaf of $\mathcal{F}_{M_{\mathcal{C} / T}^{\alpha}\left(d,\left(r_{j}^{(i)}\right)\right)}$ such that $\pi^{\prime} \circ \tilde{\gamma}=\gamma$ and that $\tilde{\gamma}(0)=x$.

Proof. Take any path $\gamma:[0,1] \rightarrow T$ and a point $x \in M_{\mathcal{C} / T}^{\boldsymbol{\alpha}}\left(d,\left(r_{j}^{(i)}\right)\right)$ such that $\pi^{\prime}(x)=\gamma(0)$. Since $p: \tilde{M}_{\mathcal{C} / T}^{\alpha}\left(d,\left(r_{j}^{(i)}\right)\right) \rightarrow M_{\mathcal{C} / T}^{\alpha}\left(d,\left(r_{j}^{(i)}\right)\right)$ is surjective, there is a point $\tilde{x} \in \tilde{M}_{\mathcal{C} / T}^{\alpha}\left(d,\left(r_{j}^{(i)}\right)\right)$ such that $p(\tilde{x})=x$. By the geometric Painlevé property stated in $\left[4\right.$, Theorem 2.3], there is a unique path $\gamma^{\prime}:[0,1] \rightarrow M_{\mathcal{C} / T}^{\boldsymbol{\alpha}^{\prime}}(\tilde{\boldsymbol{t}}, r, d)$ such that $\gamma^{\prime}(0)=$ $\tilde{x}, \pi(\tilde{x})=\gamma(0)$ and that the image of $\gamma^{\prime}$ lies in a leaf of the foliation determined by $D\left(\pi^{*}\left(\Theta_{T}\right)\right) \subset \Theta_{M_{\mathcal{C} / T}^{\alpha^{\prime}}(\tilde{\boldsymbol{t}}, r, d)}$. By construction, the image of $\gamma^{\prime}$ in fact lies in $\tilde{M}_{\mathcal{C} / T}^{\alpha}\left(d,\left(r_{j}^{(i)}\right)\right)$. So the path $p \circ \gamma^{\prime}$ satisfies the desired condition.

REMARK 4.1. As in Remark 2.1, there is an analytic isomorphism from the moduli space of stable parabolic connections with given spectral type to the moduli space of stable filtered local systems given by Yamakawa. By extending this Riemann-Hilbert analytic isomorphism to the isomorphism between the relative moduli spaces over $T$, we can also obtain the geometric Painlevé property.

\section{References}

[1] W. Crawley-Boevey, Indecomposable parabolic bundles and the existence of matrices in prescribed conjugacy class closures with product equal to the identity, Publ. Math. Inst. Hautes Études Sci., 100 (2004), 171-207.

[ 2 ] K. Fuji and T. Suzuki, Drinfeld-Sokolov hierarchies of type A and fourth order Painlevé system, Funkcial. Ekvac., 53 (2010), 143-167.

[3] K. Fuji and T. Suzuki, Higher order Painlevé system of type $D_{2 n+2}^{(1)}$ arising from integrable hierarchy, Int. Math. Res. Not. IMRN, 2008 (2008), Art.ID rnm129.

[ 4 ] M. Inaba, Moduli of parabolic connections on a curve and Riemann-Hilbert correspondence, J. Algebraic Geom., 22 (2013), 407-480.

[ 5 ] M. Inaba, K. Iwasaki and M.-H. Saito, Moduli of stable parabolic connections, Riemann-Hilbert correspondence and geometry of painlevé equation of type VI, Part I, Publ. Res. Inst. Math. Sci., 42 (2006), 987-1089.

[6] M. Inaba, K. Iwasaki and M.-H. Saito, Dynamics of the sixth Pailevé Equations, in Théories asymptotiques et équations de Painlevé, Sémin. Congr., 14, Soc. Math. France, Paris, 2006, 103-167.

[ 7 ] H. Kawakami, A. Nakamura and H. Sakai, Degeneration scheme of 4-dimensional Painlevé equations, arXiv:1209.3836.

[ 8 ] H. Kawakami, A. Nakamura and H. Sakai, Toward a classification of four-dimensional Painlevétype equations, Algebraic and geometric aspects of integrable systems and random matrices, Contemp. Math., 593, Amer. Math. Soc., Providence, RI, 2013, 143-161.

[ 9 ] T. Oshima, Classification of Fuchsian systems and their connection problem, Exact WKB analysis 
and microlocal analysis, RIMS Kôkyûroku Bessatsu, B37, Res. Inst. Math. Sci. (RIMS), Kyoto, 2013, arXiv:0811.2916v2, 163-192.

[10] M.-H. Saito and S. Szabo, Apparent singularities and canonical coordinates for moduli of parabolic connections and parabolic Higgs bundles, in preparation.

[11] H. Sakai, Isomonodromic deformation and 4-dimensional Painlevé type equations, preprint, University of Tokyo, Mathematical Sciences, 2010.

[12] Y. Sasano, Coupled Painlevé VI systems in dimension four with affine Weyl group symmetry of type $D_{6}^{(1)}$, II, RIMS Kôkyûroku Bessatsu B5, 2008, 137-152.

[13] D. Yamakawa, Geometry of multiplicative preprojective algebra, Int. Math. Res. Pap. IMRP 2008, Art. ID rpn008, 77pp. (arXiv:0710.10.2649v4 [math.SG] 15 Nov 2007)

Michi-aki InABA

Department of Mathematics

Graduate School of Science

Kyoto University

Kyoto 606-8502, Japan

E-mail: inaba@math.kyoto-u.ac.jp

\section{Masa-Hiko SAITo}

Department of Mathematics Graduate School of Science

Kobe University

Kobe 657-8501, Japan

E-mail: mhsaito@math.kobe-u.ac.jp 